\title{
Experimental study of nonlinear interaction of plasma flow with charged thin current sheets: 2 . Hall dynamics, mass and momentum transfer
}

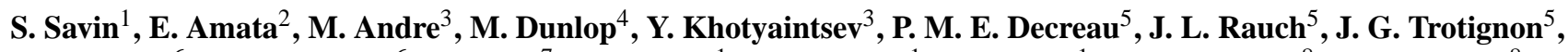 \\ J. Buechner ${ }^{6}$, B. Nikutowski ${ }^{6}$, J. Blecki ${ }^{7}$, A. Skalsky ${ }^{1}$, S. Romanov ${ }^{1}$, L. Zelenyi ${ }^{1}$, A. M. Buckley ${ }^{8}$, T. D. Carozzi ${ }^{8}$, \\ M. P. Gough ${ }^{8}$, P. Song ${ }^{9}$, H. Reme ${ }^{10}$, A. Volosevich ${ }^{11}$, H. Alleyne ${ }^{12}$, and E. Panov ${ }^{6,1}$ \\ ${ }^{1}$ Space Research Institute, 117997, Profsoyuznaya 84/32, Moscow, Russia \\ ${ }^{2}$ Istituto di Fisica dello Spazio Interplanetario, INAF, Roma, Italy \\ ${ }^{3}$ Swedish Institute of Space Physics, Uppsala, Sweden \\ ${ }^{4}$ Space Science and Technology Department, Rutherford Appleton Laboratory, UK \\ ${ }^{5}$ Laboratoire de Physique et Chimie, de l'Environnement, CNRS, Orléans, France, France \\ ${ }^{6}$ Max-Planck-Institut fur Sonnensystemforschung, Katlenburg-Lindau, Germany \\ ${ }^{7}$ Space Research Center, Polish Academy of Sciences, Warsaw, Poland \\ ${ }^{8}$ Space Science Centre, University of Sussex, UK \\ ${ }^{9}$ University of Massachusetts, Lowell, USA \\ ${ }^{10}$ Centre d'Etude Spatiale de Rayonnement, CNRS/UPS/OMP, Toulouse, France \\ ${ }^{11}$ Mogilev State University, Belarus \\ ${ }^{12}$ Control and Systems Engineering, University of Sheffield, UK
}

Received: 3 May 2005 - Revised: 23 March 2006 - Accepted: 23 March 2006 - Published: 10 August 2006

Part of Special Issue "Nonlinear and multiscale phenomena in space plasmas"

\begin{abstract}
Proceeding with the analysis of Amata et al. (2005), we suggest that the general feature for the local transport at a thin magnetopause (MP) consists of the penetration of ions from the magnetosheath with gyroradius larger than the MP width, and that, in crossing it, the transverse potential difference at the thin current sheet (TCS) is acquired by these ions, providing a field-particle energy exchange without parallel electric fields. It is suggested that a part of the surface charge is self-consistently produced by deflection of ions in the course of inertial drift in the nonuniform electric field at MP.

Consideration of the partial moments of ions with different energies demonstrates that the protons having gyroradii of roughly the same size or larger than the MP width carry fluxes normal to MP that are about $20 \%$ of the total flow in the plasma jet under MP. This is close to the excess of the ion transverse velocity over the cross-field drift speed in the plasma flow just inside MP (Amata et al., 2005), which conforms to the contribution of the finite-gyroradius inflow across MP. A linkage through the TCS between different plasmas results from the momentum conservation of the
\end{abstract}

Correspondence to: S. Savin

(ssavin@iki.rssi.ru) higher-energy ions. If the finite-gyroradius penetration occurs along the MP over $\sim 1.5 R_{E}$ from the observation site, then it can completely account for the formation of the jet under the MP. To provide the downstream acceleration of the flow near the MP via the cross-field drift, the weak magnetic field is suggested to rotate from its nearly parallel direction to the unperturbed flow toward being almost perpendicular to the accelerated flow near the MP.

We discuss a deceleration of the higher-energy ions in the MP normal direction due to the interaction with finite-scale electric field bursts in the magnetosheath flow frame, equivalent to collisions, providing a charge separation. These effective collisions, with a nonlinear frequency proxy of the order of the proton cyclotron one, in extended turbulent zones are a promising alternative in place of the usual parallel electric fields invoked in the macro-reconnection scenarios. Further cascading towards electron scales is supposed to be due to unstable parallel electron currents, which neutralize the potential differences, either resulted from the ion- burst interactions or from the inertial drift.

The complicated MP shape suggests its systematic velocity departure from the local normal towards the average one, inferring domination for the MP movement of the non-local processes over the small-scale local ones.

Published by Copernicus GmbH on behalf of the European Geosciences Union and the American Geophysical Union. 
The measured Poynting vector indicates energy transmission from the MP into the upstream region with the waves triggering impulsive downstream flows, providing an input into the local flow balance and the outward movement of the MP.

Equating the transverse electric field inside the MP TCS by the Hall term in the Ohm's law implies a separation of the different plasmas primarily by the Hall current, driven by the respective part of the TCS surface charge. The Hall dynamics of TCS can operate either without or as a part of a macro-reconnection with the magnetic field annihilation.

\section{Introduction}

We proceed with analysis of the transport processes and mechanisms for plasma jet acceleration at a thin current sheet (TCS, $\sim 90 \mathrm{~km}$ thick) observed on 13 February 2001, at $\sim 20: 01 \mathrm{UT}$, which has been identified with the magnetopause (MP) in the companion paper Amata et al. (2005), cited as $[\mathrm{A}]$ hereafter, where the Cluster data used and the respective instrumentation are described. Via the ion finitegyroradius effect, the TCS with a potential jump $\sim 300 \mathrm{~V}$ provides a partial mass and momentum transfer and energy conversion [A]. From 3 timing differences between the electric field measurements from all Clusters at MP, on a 2min time scale, a constant MP velocity is found $V_{\mathrm{MP}}=(26.2$; $0.2 ;-15.7) \mathrm{km} / \mathrm{s}$ in GSE, while MP normals from minimum variance analysis of magnetic field yields $N_{B}=(0.96,-0.21$, -0.16 ) at $\sim 25^{\circ}$ from $V_{\text {MP }}$. At shorter time scales of $\sim 10 \mathrm{~s}$ at the MP the constant-velocity (CVA, see Haaland et al., 2004) and minimum variance approaches are inconsistent with ion low-energy cutoffs. Maximum variance analysis for the electric field gives common normal $N_{E}=(0.95,-0.3,0.087)$, which is at $16^{\circ}$ to the $N_{B}$, and at $40^{\circ}$ the $V_{\mathrm{MP}}$. Constant MP thickness approach (CTA, see Haaland et al., 2004) gives a normal $N_{\mathrm{CTA}} \sim(0.815,0.579,0.02)$ and speeds for Cluster $1,2,3,4: V_{\mathrm{CTA}} \sim 41 ; 35 ; 43 ; 33 \mathrm{~km} / \mathrm{s}$. The average $V_{\mathrm{CTA}}$ magnitude lies in a $21 \%$ window from $V_{\mathrm{MP}}[\mathrm{A}]$.

In this paper we propose an explanation for the different directions of the local normal from both minimum variance of the magnetic field and from maximum variance of the electric field versus direction of the MP motion from the observed time delays on 4 spacecraft (cf. Dunlop et al., 2005), suggesting that the motion of an indented MP is defined by non-local properties of the near-cusp region as an entity. We compare the difference between ion velocity and the crossfield drift velocity, demonstrating a systematic deficit of the drift speed of $\sim 25 \%$ only in the tailward plasma jet just inside the TCS [A], with the plasma inflow in the MP normal direction due to the finite-gyroradius effect. We show the necessary extra Cluster 1 data and apply the results of multipoint analysis from [A] for the scale estimation and plasma current calculations.
The Hall dynamics of a thin MP current sheet is outlined. Previous studies of data from Interball-1, Polar and Cluster (Nikutowski et al., 1998, 1999; Mozer et al., 2003a; Vaivads et al., 2004) have been mostly limited to the comparison with models of stationary X-line macro reconnection. In the TCS the frozen-in condition is violated due to the small current scale, which can be described not only in reconnection-like approximations, but also in terms of gyroviscosity (Stasiewicz, 1994; Sibeck et al., 1999). Belmont and Rezeau (2001) use the term "micro reconnection" for similar effects of Alfven waves on the magnetic flux erosion. The Hall dynamics of the charged TCS, might include transient (and multiple) X-, Y-, O-lines etc. as particular scenarios for the current sheet evolution (see e.g. Syrovatskii, 1971; Sibeck et al., 1999; Zelenyi et al., 2002; Silin and Buechner, 2003; Savin et al., 2004b, 2005a). For a simple model Mozer et al. (2003) demonstrate that the Hall dynamics per se can explain local plasma structuring without local reconnection features (e.g. parallel electric fields and electron diffusion regions). We propose that electrons with large thermal speeds can provide surface charges at the TCS moving along field lines. The surface charges support transverse diamagnetic currents, separating different plasmas via the Hall effect (cf. Mozer et al., 2003). In turn, the potential difference for driving the field-aligned electron current might result from inertial drift of the incident (MSH) ions in the non-uniform electric field of the surface charge, seen in the frame of bulk motion by the MSH plasma; and the inertial drift results in ion separation from electrons (cf. Genot et al., 2004; Savin et al., 2004a, 2005a). Recent papers, claiming crossings of a diffusion region (e.g. Vaivads et al., 2004; Mozer et al., 2003a), in reality confirm the Hall ion dynamics in TCS and show the existence of substructures of electron-gyroradius (inertiallength) scales, the presence of which automatically implies breaking of frozen-in conditions for electrons (cf. Savin et al., 1998; Andre et al., 2004). Those features naturally are present in reconnection models, while they are also characteristic of a kinetic TCS without reconnection (see e.g. Silin and Buechner, 2003). Filamented electron beams of electron scales also account for the neutralization of the potential difference, generated by the charge separation through inertial drift (Genot et al., 2004). The inertial drift in standing electric wave-packets at the outer border of the MP boundary layer is able to accelerate structured jets up to magnetosound and highly super-Alfvenic velocities, and the jet dynamic pressure can largely exceed that of the magnetic one at a downstream MP (Savin et al., 2004a, 2005b).

In this second companion paper we proceed with the discussion of the thin (of the order of proton gyroradius) plasma separatrix between MSH and magnetospheric plasmas in boundary layers at $\sim 20: 01$ UT [A], shifting the stress towards the data interpretation in the light of plasma and momentum transport across the MP. We incorporate interpretation of the data in the MP vicinity with the inertial drift and Hall physics, and discuss it versus a reconnection input 
into the dynamics, mass and momentum balance at MP. It is demonstrated that the finite-gyroradius effect can account for a substantial mass and momentum transfer across MP. We suggest a non-reconnection mechanism for the formation of plasma streams near MP: the larger-energy ions conserve their momentum parallel to MP during the motion across MP in the high-beta plasma, resulting in the motion of the inner plasma without an annihilation of the magnetic field, i.e. without the macro reconnection, the effects of which could be superimposed or not.

\section{Plasma inflow and momentum balance}

In $[\mathrm{A}]$ it has been demonstrated that the largest flow bursts near the MP (i.e. jets, see Fig. 1 of [A] at $\sim 19: 30,19: 50$ and 20:01 UT) occur perpendicularly to the magnetic field direction in contrast to the Gas Dynamic Convection Field (GDCF) model prediction and to unperturbed nearly parallel flows (see [A] and Savin et al., 2002 for the GDCF descriptions). Thus the perpendicular flows are of general importance for the mass and momentum balance in the MP vicinity. To estimate how finite-gyroradius effects affect the flow balance near the MP, in [A] the drift velocity $V_{d}=\boldsymbol{E} \times \boldsymbol{B}$ is compared with $V_{\text {perp }}$, i.e. the ion velocity perpendicular to the magnetic field (see Fig. 8 in [A]). For the enhanced plasma flow just inside MP at 20:00:50-20:02:00 UT (called "MP jet") [A] roughly estimate that $V_{\text {perp }}$ is higher than $V_{d}$ by $25 \%$.

To facilitate further analysis of the thin nonlinear structure, TCS, we calculate the partial moments for ions with energy larger than a chosen value in the spacecraft frame. However this might pose certain errors as we cannot calculate these moments in a plasma bulk frame for two reasons: (i) the flow velocity changes much on each time step of $4 \mathrm{~s}$, while we need to compare the input in the flow from ions with different energies over the interval of several minutes; (ii) the plasma appears to be a non-equilibrium one with co-existing ion populations with different moments (see Figs. 3, 2 and 6-7 in [A] and related discussion in [A]), that, in principle, requires extracting separate moments for each ion population and a comparison with respective multi-fluid equations (planned for a future paper).

Figure 1 displays, in the top panel, the total ion number density (thin blue curve), compared with the GDCF prediction (thick gray line), along with partial ion number densities, for energies larger than $300 \mathrm{eV}$ (black) and $1 \mathrm{keV}$ (red). The bottom panel shows the $E_{x}$ electric component (green) and its GDCF predictions (dark blue line). Finally, Inserts A and $\mathrm{B}$, illustrate the rotation of the magnetic field vector in the presence of a regular difference between $E_{x}$ and its GDCF proxy. In general the total ion density follows the GDCF one until 19:50 UT, when the total density starts to fluctuate and finally drops at $\sim 20: 07$ UT toward the mantle value. At the innermost edge of the "diamagnetic cavity" before 20:07 UT

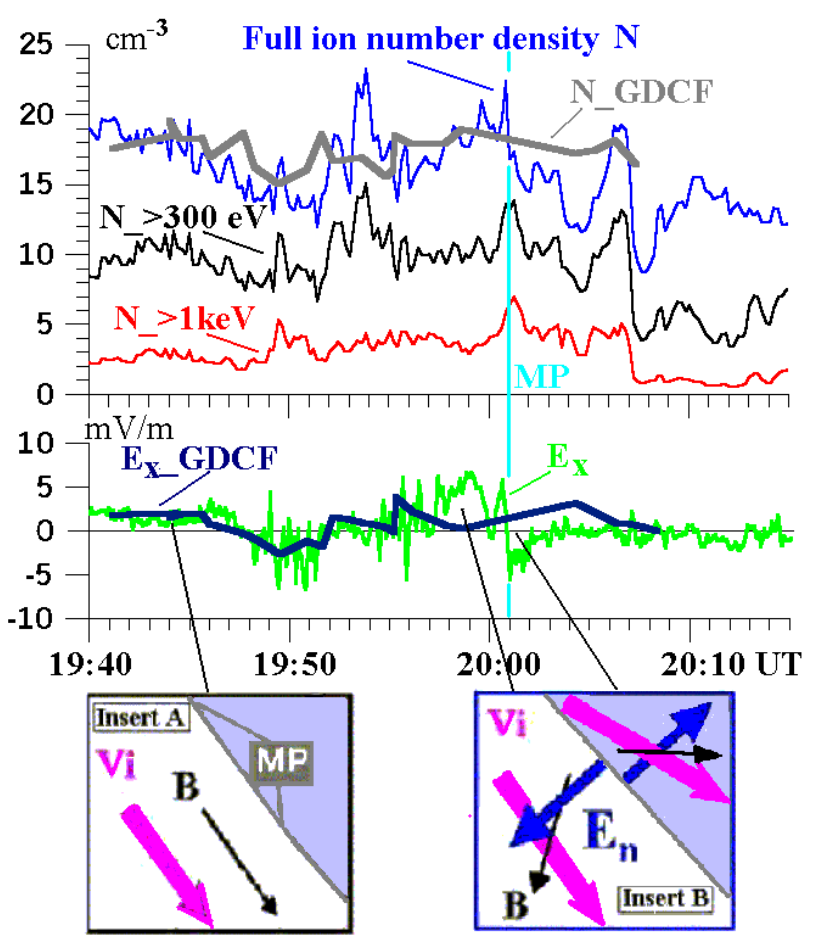

Fig. 1. 13 February 2001. Top panel: Cluster 1 ion density (blue) near the MP entry ( 20:01 UT); GDCF density (grey), Cluster 1 density for ions with energy $>300 \mathrm{eV}$ (black), $>1 \mathrm{keV}$ (red). Bottom panel: Cluster $E_{x}$ in spacecraft frame (green) and GDCF $E_{x}$ (thick blue line). Insert $\mathrm{A}-$ scheme for ion velocity $V_{i}$ (violet arrow) and magnetic field (black arrow) in unperturbed flow around MP, when the electric field in the flow frame is negligible; Insert B - scheme of the flow disturbed by the "active" DC electric field (marked by " $E_{n}$ ", blue arrows) along the MP normal. Note the rotation of the magnetic field and the acceleration of the flow.

(cf. Fig. 1 in [A]) dense cold plasma appears again, flowing now generally along field lines from an upstream outer cusp. Inside the region of density fluctuations (19:50-20:07 UT) ions of $>1 \mathrm{keV}$ are intensified, having maxima at 19:50 and 20:01 UT (cf. Fig. 1 in [A]). The latter is just inside MP, preceded by the sharp maximum of the total density outside MP, which corresponds to MSH flow, piled up by the MP outward motion at $\sim 35 \mathrm{~km} / \mathrm{s}$. Density of ions with energy $>300 \mathrm{eV}$ generally follows the total density outside MP and the density of the more energetic ions inside MP. After 19:50 UT density of ions with energy $>1 \mathrm{keV}$ grows presumably due to escaping of the ions with finite-gyroradius from the cusp [A] and to probable wave-particle interactions, which can be inferred from correlations between the density departures from GDCF and fluctuations of $E_{x}$ (green line). The higherenergy density further grows inside the "plasma ball" (i.e. large-scale $|B|$ depletion at 20:01-20:07 UT in Fig. 1 [A], see also Savin et al. (2005a, b) dropping in the mantle after 20:07 UT, where cold ions dominate (see the difference between blue and black curves in [A], Fig. 5, and related discussion below). 

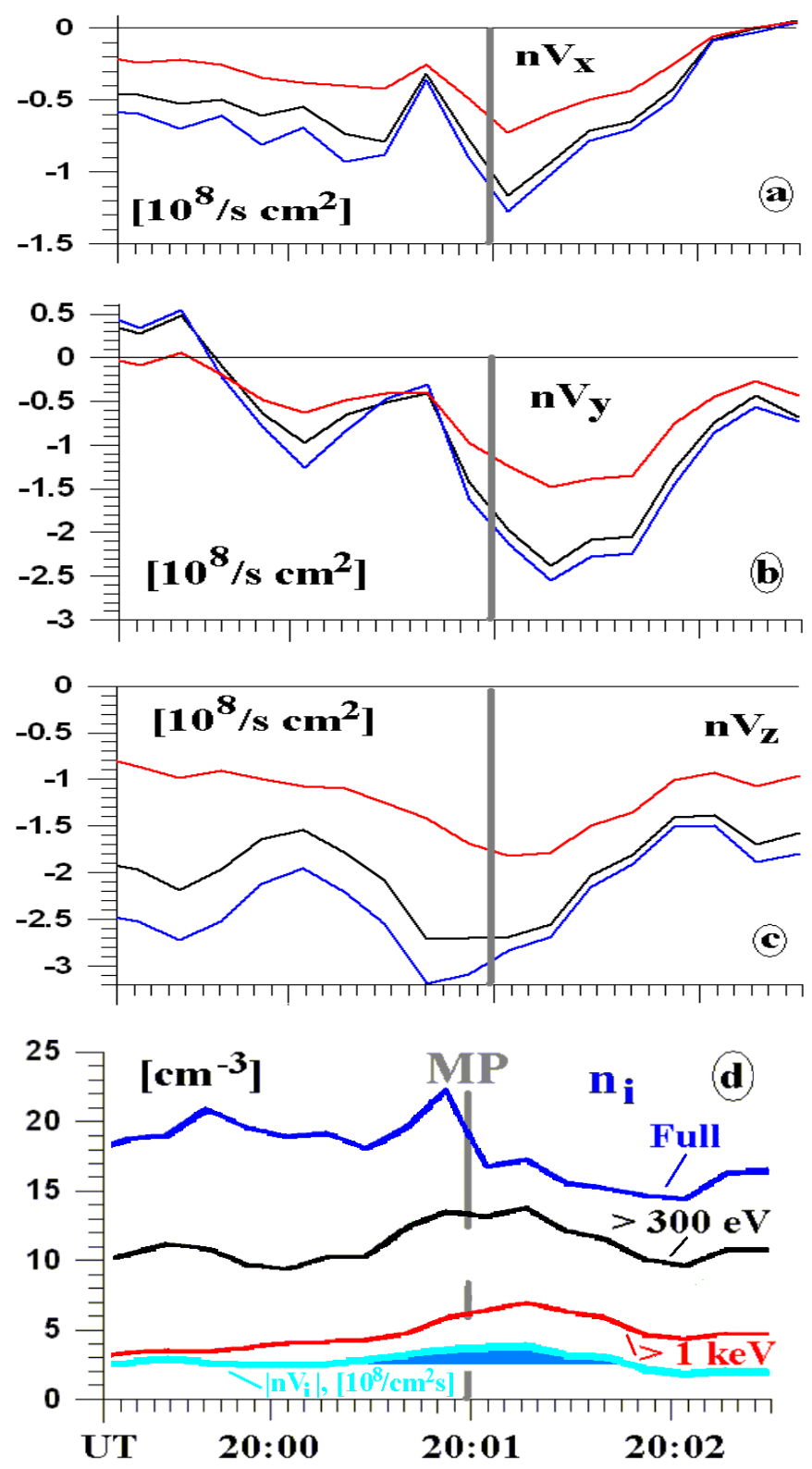

Fig. 2. Cluster-1, 13 February 2001. (a) ion flux " $n V_{i x}$ ", blue lines - full CIS energy range (see [A]), black - partial ion flux for $>300 \mathrm{eV}$, red - for $>1 \mathrm{keV}$ ions; (b) the same for " $n V_{i y}$ "; (c) the same for " $n V_{i z}$ "; (d): ion density $n_{i}$ (blue), partial ion density for energies $>300 \mathrm{eV}$ (black), that of $>1 \mathrm{keV}$ (red) and full ion flux (cyan line, the jet is marked by shadowing, see text for details).

Insert A of Fig. 1 shows the mutual orientation of the ion flow velocity $V_{i}$ (violet), magnetic field $B$ (black) and MP in the unperturbed MSH flow: $V_{i}$ and $B$ are nearly parallel, conforming to the GDCF model predictions. Insert B shows the actual situation in the case under study. The presence of the bipolar normal electric field $E_{n}$ (blue) results mainly in the $B$ rotation and in the $V_{i}$ rise due to the cross-field drift (in case of Insert $A$ the flow acceleration by $E_{n}$ is impossible in the shown plane). Thus, the rise of the downstream flow velocity described in Fig. 4 of [A] (cf. also Fig. 4 herein) should be the consequence not only of $E_{n}$, but also of the forced magnetic field rotation. Namely this magnetic field rotation makes the flow transverse, at $~ 19: 56-19: 58$ and 20:0120:02 UT (see 3rd panel from bottom in Fig. 1 of [A]).

Figure 2 displays, in the bottom panel, the CIS-HIA proton number density (blue line) and the number density for protons with energy higher than $300 \mathrm{eV}$ and $1 \mathrm{keV}$ (black and red line respectively). The last curve in the bottom panel shows the total proton flux (cyan line). The remaining panels display the three components of proton flux, calculated for the full distribution (blue line) and for energies greater $300 \mathrm{eV}$ and $1 \mathrm{keV}$ (black and red lines respectively). The maximum of the lower-energy ions, which are reflected by the MP TCS (see Fig. 2 in [A]), at $~ 20: 00: 53$ UT infers placing their source outside the MP. In this region the ion flux is carried by the colder ions, as one can see in panels a-c from the difference between the blue and black curves. The large-gyroradius ions (energy $>1 \mathrm{keV}$, red lines) carry up to $60 \%$ of the flux in the jet inside MP. Note inward largegyroradius flow $n V_{i x}$ (panel a), which constitutes $\sim 20 \%$ of the total flow in the inner jet. The ions, carrying this inward flux, should not feel the small-scale $(<$ their gyroradius) MP current sheet, i.e. the higher-energy flux gives the lower-limit estimate for the local influx. The intermediateenergy ions (marked black) could also provide an input into the inflow, while the minimum influx at 20:00:54 UT is defined largely by the large-gyroradius influx. At the magnetospheric border of the inner jet (after 20:02 UT) there is a vanishing $\mathrm{X}$-component of inflow (the normal from magnetic field minimum variance $N_{B}$ is close to $\mathrm{X}$, see [A]). Thus from the necessary mass-conservation in an almost incompressible plasma, we suggest that this inflow provides a local source for the jet inside the MP. Its minimum contribution (estimated above as $\sim 20 \%$ ) agrees fairly well with $\sim 25 \%$ of the ion velocity ( $V_{\text {perp }}$ ) excess over the electric-drift velocity $\left(V_{d}\right)$ in Fig. 8 from [A]. Taking the estimate for width of the inner plasma jet $\sim 2000 \mathrm{~km}$ (cf. [A]), one obtains a proxy for the scale along MP of $\sim 1.5 R_{E}$, at which the jet can be formed uniquely by the inflow from finite-gyroradius effects (providing similar MP structure upstream from the Cluster crossing). The $1.5 R_{E}$ is almost one half of the characteristic dimension along MP of "plasma balls" (Savin et al., 2005a); thus we regard the finite-gyroradius effect as a major plasma source for the inner jet. An interesting feature in Fig. 2, is the conservation of the dominant $n V_{z}$ flux (panel c) across the MP for ions with energy $>300 \mathrm{eV}$. These protons with gyroradius of roughly the same size or larger than the MP width carry the major ion flux on both sides of the MP.

We make now use of the time-of-flight effect to estimate the distance of a remote source for the protons with lowenergy cutoff, which is suggestive for the finite gyroradius leakage of ions from the outer cusp. For that purpose, 


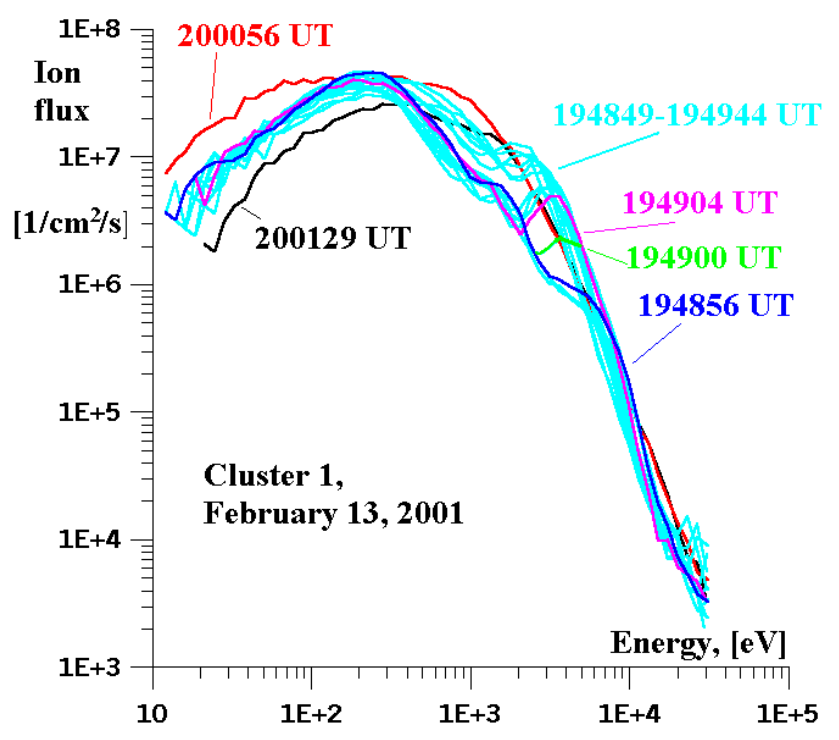

Fig. 3. 4 s resolution CIS 2 omni-directional differential fluxes as a function of energy, Cluster 1, 13 February 2001 See text for details).

Fig. 3 displays the omni-directional Cluster 1 spectra from 19:48:49 to 19:49:44 UT from the leading edge of an event of departure of electric field, clock-angle and $B_{y}$ from the GDCF predictions at $\sim 19: 50$ UT (see Fig. 1 and Fig. 1 of [A]). For reference, we plotted a spectrum measured just ahead the MP TCS (in red, marked by "200056 UT", see Fig. 4 in [A]) and a characteristic spectrum inside the jet at 20:01:29 UT (in black). Inspection of 3D ion distributions (not shown) shows that higher-energy protons arrive parallel to the magnetic field, i.e. from an upstream region along the MP. We marked by different colors 3 consecutive spectra at 19:48:56, 19:49:04 and 19:49:04 UT, which we use to estimate the distance from a source of heated proton using time-of-flight methodics (see e.g. Sibeck et al., 1999).

This yields a distance of $2-3 R_{E}$, which implies that the plasma source is at a side of the cusp throat rather than at the position of the dayside anti-parallel magnetic fields across MP, where the large-scale reconnection should occur for the measured southward IMF $\left(\sim 5-6 R_{E}\right.$, see Fig. 6 and related discussions below, cf. Sibeck et al., 1999, and Cargill et al., 2004). We attribute the well seen higher-energy maximum in the remaining spectra from the interval 19:49:0419:49:44 UT to the penetration of the heated ions from the boundary layer inside cusp due to the finite-gyroradius effect (cf. Figs. 4, 6 in [A]). In such case the MP width might be remotely sounded along field lines: at about 19:49:04 UT the low-energy cutoff is $\sim 3 \mathrm{keV}$ that corresponds to a MP thickness of $\sim 190 \mathrm{~km}$ (provided the similar strength and profile of magnetic field at MP); then the cutoff energy evolves towards $2 \mathrm{keV}$, which suggests a MP width of $\sim 160 \mathrm{~km}$. Thus, the estimated distance from the ion leakage event suggests widespread ion-gyroradius effect that in turn is a sign of its
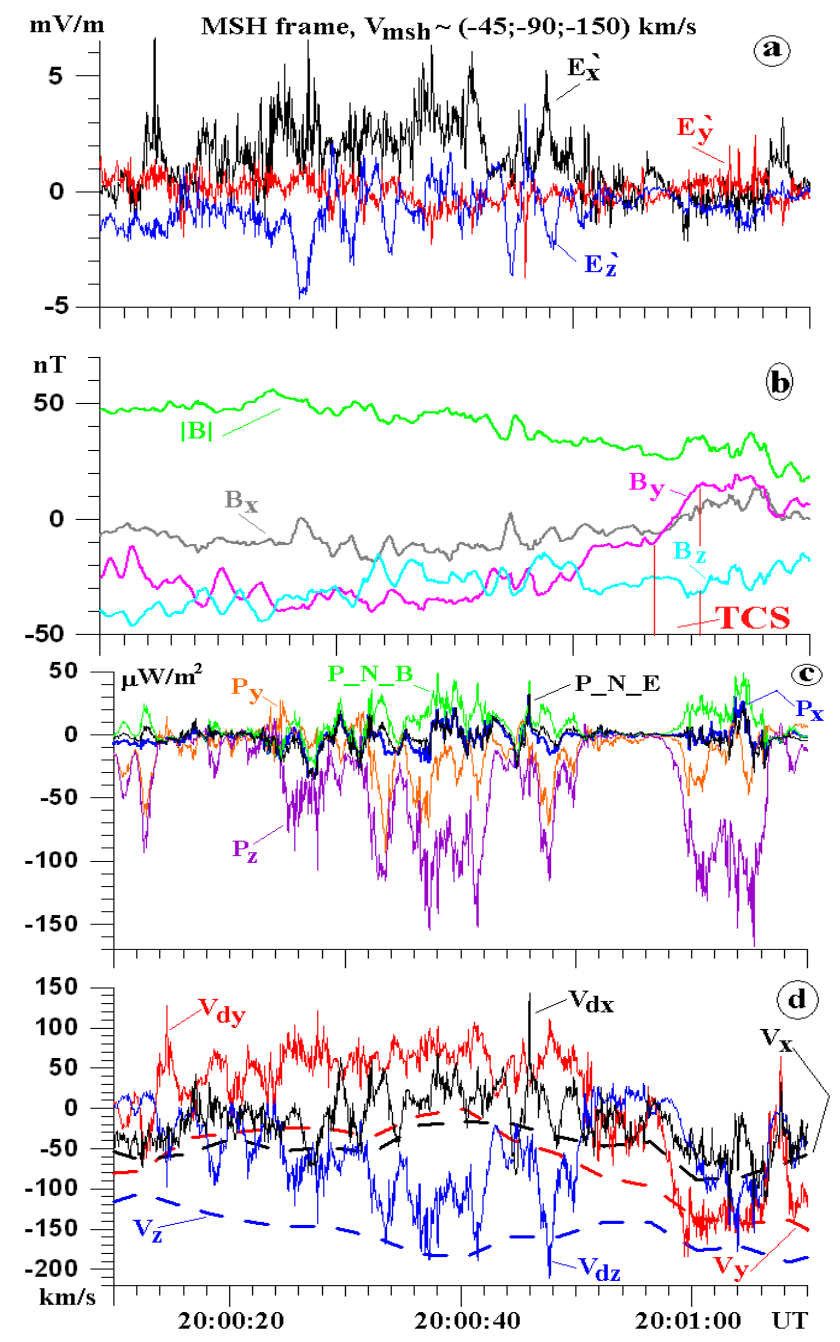

Fig. 4. Cluster-1, 13 February 2001. (a) GSE vector of electric field $\left(E^{\prime}\right)$ in a MSH-flow frame with $V_{\mathrm{MSH}}=(-45,-90,-150) \mathrm{km} / \mathrm{s}$ in front of the TCS (marked by 2 vertical lines in panel b); (b) magnetic field vector and magnitude; (c) Poynting flux $(\boldsymbol{P})$ GSE components in spacecraft frame, calculated from electric and magnetic field cross-product after subtracting of signal, averaged by the spline procedure with 2001 points of the interpolated data with sampling at $25 \mathrm{~Hz}$; P_N_B green line - Poynting flux along the average normal from magnetic minimum variance analysis $N_{B}, \mathrm{P} \_\mathrm{N} \_\mathrm{E}-$ the same along direction electric field of maximum variance $N_{E} ;(\mathbf{d})$ GSE electric drift velocity $\left(V_{d}\right)$ and ion velocity ( $V$, dashed line) in spacecraft frame.

importance for the plasma transfer and jet formation. However, we should mention that bursty reconnection at the cusp MP (see e.g. Savin et al., 2004b) could also provide the leakage of the heated MSH-like plasma from the cusp. But in this case the question arises, why the outcoming population displays only low-energy cutoff without any detectable acceleration and heating? One more option is to treat this population as a MSH one, "reflected" from a reconnecting MP; however, for such an approach neither energy source nor mechanism, 
defining the coefficient of reflection for the ions being able to cross a finite-gyroradius MP, have been introduced (see Sibeck et al., 1999).

We now consider the contributions of $\mathrm{AC}$ and quasi DC electric fields to the flow balance. In Fig. 4 we present GSE vectors of the electric field from Cluster 1 (panel a) in a MSH-flow frame, moving at a characteristic speed $V_{\mathrm{MSH}}=(-45,-90,-150) \mathrm{km} / \mathrm{s}$ near the MP (see Fig. 1 in [A], cf. Savin et al., 2004a); of magnetic field (panel b); of Poynting flux (panel c, spacecraft frame); of ion velocity and of electric drift velocity (panel d, spacecraft frame). Further in the text we use the notation $E^{\prime}$ for the electric field components in the MSH frame. The unperturbed $E_{x}^{\prime}$ and $E_{y}^{\prime}$ at the left side of Fig. 4 are close to zero, inferring for these components that the chosen particular value of $V_{\mathrm{MSH}}$ is a good proxy for transformation into the MSH-flow frame for the total time interval; $E_{z}^{\prime}$ at the left side of Fig. 4 has a DC offset, requiring a corresponding change of the MSH speed for the data transformation at the time interval beginning, but we neglect this offset relative to the strong disturbances, visible at 20:00:30-20:00:50 UT in Fig. 4, generally in $E_{x}^{\prime}$. So, the transformation into a MSH frame demonstrates that the incident ions are affected mainly by the $E_{x}^{\prime}$ component, which is close to the MP normal (see Introduction and discussion in [A]). Note that electric field at the TCS is diminished in this frame, but the transformation used has no explicit sense under MP, which partially reflects the incident flow and partially transfers it (see discussion of Figs. 2 and 4-7 in [A]).

The main input of the quasi DC electric field into the flow balance near the MP is an acceleration of the plasma downstream, mainly in the $-\mathrm{Z}$ direction in front of the MP, via the cross-field drift (see panel d). The strong positive $E_{x}^{\prime}$ disturbances have a local origin, in contrast to the global GDCF predictions (see Fig. 1 - green line versus thick violet trace, cf. [A]). The similarity of profiles of $V_{d z}$ and Poynting flux $P_{z}$ component indicates that waves accelerate the plasma in the same direction at 20:00:25-20:00:50 UT, presumably via inertial drift (see Eq. (2) below). Averaging $V_{d z}$ over the interval of maximum drift (20:00:25-20:00:50 UT) gives $91 \mathrm{~km} / \mathrm{s}$ with a standard deviation of $39 \mathrm{~km} / \mathrm{s}$. As this averaging includes the input of the AC spikes, we attribute $\sim 52 \mathrm{~km} / \mathrm{s}$ to the average cross-field drift, i.e. $>1 / 3$ of the accelerated flow should in this case be due to the $\mathrm{AC}$ input. We do the crude dividing of $\mathrm{AC}$ and DC components by estimating of average input from the waves into $V_{d z}$ similar to $P_{z}$ : its mean value is comparable with its deviation. The positive projections of Poynting flux onto the normals (pointed sunward) and positive $P_{x}$ - spikes demonstrate that the waves propagate upstream, being most probably reflected from the MP (cf. Belmont and Rezeau, 2001 and Savin et al., 2005a) or emitted by the jet. $V_{d y}$ outside MP is pointed in the $+\mathrm{Y}$ direction (i.e. along the smooth MP, see Fig. 5 and related discussion below), while inside MP it is along -Y-axis, presumably due to the opposite normal electric field from different MP sides. It appears strange that the AC disturbances $\left(P_{y}\right)$ outside MP propagate in the opposite direction relative to the main outer $V_{d y}$, i.e. along -Y-axis, as the waves inside MP do, and a correlation of the latter with $V_{y}$ (dashed line) points to transport of the inner disturbances downstream by the jet. As the inner disturbances have the positive $P_{x}$ and normal components, they should represent a source for the outer ones. Another reason for the outlined behavior would be guiding of the smaller-scale disturbances by the cusp throat, which has the opposite curvature compared to that of the smooth MP (see Fig. 5 and discussions below). We have checked that the Poynting flux and drift velocity behave similarly to that of Fig. 4 at 20:00:32-20:00:50 UT since 19:56 UT, i.e. when $E_{x}$ departs from the GDCF predictions (see Fig. 1).

\section{MP shape, motion and charging}

In this section we discuss possible applications and consequences of finite-gyroradius effects and the local DC and AC electric fields, described above, to transport processes and MP dynamics.

The picture of the global configuration on 13 February 2001 at negative (anti-sunward) magnetic dipole tilt is rather typical with the MP separating MSH and heated plasma in outer cusp, the denser higher-energy plasma at 20:0120:07 UT in Fig. 1 corresponds to "plasma ball" of Savin et al. $(2002,2005 a)$. The strong fluctuations between stagnant MSH and outer cusp in the cases of Savin et al. (2001) isolate them, while in our case a "plasma ball" represents the interior large-scale reservoir for magnetospheric plasma. First of all, it should be remembered that at the high-latitude MP we often deal with interaction of a high-beta incident plasma with a higher-beta one (cf. Savin et al., 2004a, b, 2005a, b; Lavraud et al., 2002, [A]). Thus, the magnetic forces are small and in a collisionless plasma the general plasma-plasma interactions involve electric fields, either (quasi) DC or AC. In the fortunate case under study, the DC electric field in front of MP clearly differs from the normal electric field, which would be induced by the equilibrium gasdynamic flow (i.e. predicted by the GDCF model).

A reason for the electric field departure from the model one should be the MP deformation compared to the smooth MP, symmetric relative to the Sun-Earth axis, of the GDCF model. This is illustrated by the departure from the model normal at a smooth MP (first noted by Cargill et al., 2004) $N_{\text {mod }}=(0.6,0.15,-0.75)$ of both the average normal, deduced either from minimum variance analysis of magnetic field $N_{B}$ or from the direction of maximum electric-field variance $N_{E}$, strongly towards the $\mathrm{X}$-axis. We verified that the minimum-variance direction on Cluster 3 on 200-s intervals departs from the $N_{B 3} \sim(0.97 ;-0.19 ;-0.15)$ (Cluster 3 normal from minimum variance analysis at 19:58:4720:02:07 UT, cf. [A]) by less than 25 degrees since 19:42 UT that can be accounted for stable control of the flow by the indented MP throughout this time interval, i.e. the MP indenta- 
tion is rather a stationary feature and the direction of $N_{B 3}$ is not due to a temporal surface wave (cf. Dunlop et al., 2005; Cargill et al., 2004; Savin et al., 1998, 2004b, 2005b). We illustrate the indented MP geometry in the Insert A of Fig. 5, where the deflected $N_{B 3}$ corresponds to the $3 \mathrm{~d}$ and 4 th blue vectors from the bottom. Returning to the normal electric field, inside the MP indentation the flow should be decelerated in the $-X$ direction, but could be accelerated in the $-Z$ direction to provide an equilibrium plasma outflow from the indentation (cf. Figs. 3 and 5 in Wang et al., 2004). The enlarged (reduced) $E_{x}^{\prime}$ near the MP could serve to balance the upstream inflow by extra acceleration (deceleration) of the plasma downstream.

As mentioned above, another reason for the local DC normal electric field is the outward movement of MP and boundary layers at velocities of $\sim 30-40 \mathrm{~km} / \mathrm{s}$, so that the MP, moving sunward, could remove an upstream plasma by accelerating it along the MP downstream by means of an extra normal electric field. Figure 4 confirms this suggestion by demonstrating the dominant negative component $V_{d z}$ of the cross-field velocity (cf. Fig. 5); also seen is the positive drift in the $+Y$ direction, which is probably a by-product of the normal electric field, dependent on the IMF direction. In Fig. 4 at 20:00:32-20:00:50 UT the drift quasi DC component constitutes $\sim 70 \%$ of the ion flow and $>80 \%$ of the flow at 20:00:10 UT, i.e. the DC cross-field drift constitutes the major part of the flow in front of the moving MP. Just inside MP in the jet (at 20:01-20:02 UT) $V_{d}$ constitutes $64 \%$ of the total ion speed, $V_{d y}$ being the dominant component.

We have discussed in [A] that the largest ion flow bursts near the MP are mostly perpendicular in contrast to the GDCF prediction and to the unperturbed MSH flows (see Fig. 1 [A]). This suggests an "active" role for the normal electric field in the MP vicinity in the regulation of the plasma flow balance. Remembering that the magnetic energy density is low, we propose the following scenario: the magnetic field deflection in the outer MP boundary layer results from the interaction of incident flow, streamlining around the high-beta obstacle ("pre-existed" plasma in Lavraud et al., 2002 or "plasma ball" in Savin et al., 2004a, 2005a, b), with the normal electric field, supposed to be produced by the surface charge(s) at the MP current sheet(s). It implies that in the high-beta interaction a weak magnetic field should fit to the "external" flow and "external" electric field, in contrast to the flow interaction with a magnetic barrier (with dominating magnetic pressure) at lower latitudes, where the normal electric field is generally induced by the external flow at the fixed MP current sheet (i.e. "external" magnetic field) to fit the Maxwellian boundary conditions. Those "active" electric fields should result in extra re-configuration of inner and outer boundary layers for both high- and low-beta obstacles. As in our case outside the MP the ion beta is not very high $(\sim 1.5-2)$, in the first approximation the stress of deformed magnetic field lines (i.e. a non-local factor) should be taken into account (cf. Dunlop et al., 2005).

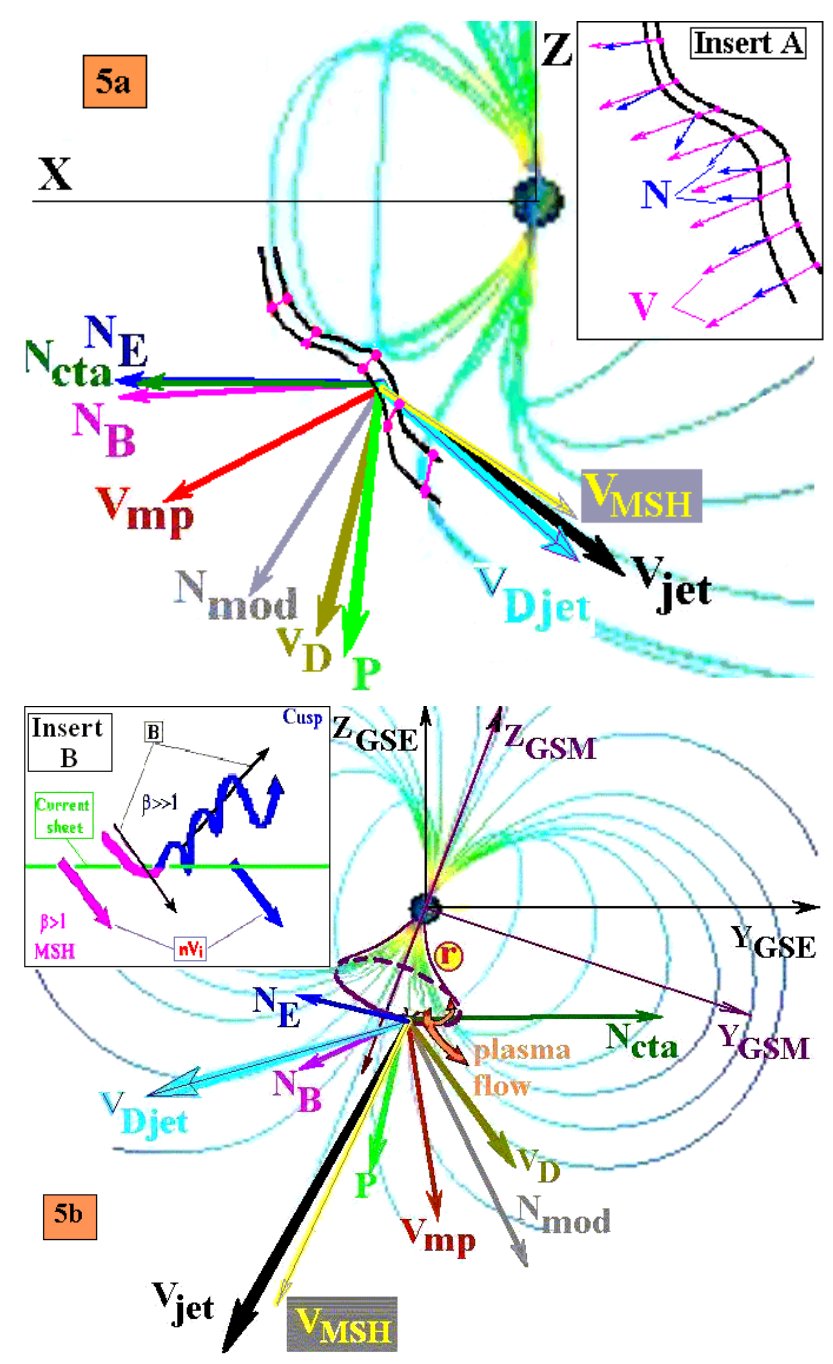

Fig. 5. Model magnetic field lines and direction of different vectors in the MP vicinity (see text) for the Cluster inbound crossing of 13 February 2001: (a) XZ GSE plane; (b) YZ GSE plane (Z and Y in GSM are superimposed). Insert A: a cartoon for MP deformation (see details in the text). Insert B: Scheme for conservation of momentum $\left(\sim n V_{i}\right.$, shown by thick arrows) by the ions with gyroradius larger than the MP width (see details in the text).

Figure 4 shows both quasi DC and $\mathrm{AC} E_{x}^{\prime}$ and $V_{d}$. While the DC/AC separation depends on the chosen upper frequency for the "DC" processes, we believe that the evaluation, made in the previous section can be utilized for an order-of-magnitude estimate. Attributing the drift spikes to waves conforms to similar shapes of the dominant $V_{d z}$ and $P_{z}$ at 20:00:32-20:00:50 UT in Fig. 4 (i.e. the spikes of drift flow are not driven by cross-terms of DC and AC components in the fields' cross-product). Thus, the AC input to the mass balance is secondary, but still substantial.

The difference outlined above in the sign of Y-components of the DC flow $\left(V_{d}\right)$ and wave propagation (Poynting vector $\boldsymbol{P}$ ) in Fig. 4 at 20:00:32-20:00:50 UT, indicates their in- 
herent different physical nature. Positive $\mathrm{P} \_\mathrm{N} \_\mathrm{B}$ (Pointing flux along the medium-scale normal from magnetic minimum variance analysis) in similar AC disturbances inside the jet under MP at 20:01:00-20:01:06 UT implies that the jet is a source for the waves in front of the MP, provided that the similar wave pattern holds in the upstream cusp (as $P_{z}>P_{x}$, cf. the above discussion of the $n V_{i x}$ role in Fig. 2). Fluctuations of the surface charge and surface waves at the TCS represent other possible sources, which are defined by the local shape of cusp throat, i.e. the sources should be of small scale (i.e. of comparable scale with that of local curvature of the cusp throat).

Fig. 5 illustrates the cusp throat configuration for the case under study in the xz GSM plane (panel a) and yz GSM plane (panel b). The geomagnetic field lines are plotted through OVT (Orbit Visualization Tool-for CLUSTER, http: //ovt.irfu.se/). The two black lines overplotted in panel a represent two probable MP intersections with the xz plane during the MP outward motion around 20:00:58 UT. The $\mathrm{xz}$ projections of a number of vectors at the Cluster position are also shown and described hereafter. In panel $b$, the cusp throat is sketched as a violet ellipse and the yz projections are plotted for the same vectors shown in panel a; moreover, the inferred plasma flow in the vicinity of the Cluster MP crossing (marked as "plasma flow") is indicated as bifurcated into tailward streaming plasma (larger arrow) and plasma entering the cusp throat (smaller arrow); finally, the possible location of anti-parallel large-scale reconnection at the dayside MP for the measured southward IMF is marked by the circled " $\mathrm{r}$ ". Insert A, which displays a blow up of the MP motion, is described in detail further on. Insert B portrays the momentum conservation across the MP. In the high-beta situation, ions crossing the MP due to their finitegyroradius should nearly conserve their momentum parallel to MP (which is supported by the $n V_{z}$ conservation across the MP for ions with energy $>300 \mathrm{eV}$ ). Just ahead of the MP the parallel flow dominates (see Fig. 2 and Fig. 1-2 of [A]), and its higher energy constituent does not feel the TCS, while the flow becomes perpendicular inside the MP. While "jumping" from MSH (over the figure plane, violet color of the ion trace), magnetic field (thin arrows) is approximately parallel to $n V_{i}$ into cusp (under the figure plane, blue color of the spiral ion trace) the ions rathly conserve their momentum (for ion $\beta>1$ ) and start to rotate in nearly perpendicular inner field. As a result, the magnetic field in the cusp with "weak" energy density should be transported by the penetrated ions, i.e. in the cusp frame respective electric field will be generated to provide respective cross-field drift (see details in text). As $\beta>>1$, this flow convects the inner magnetic field along the MP tailward and results in a contribution into the convective electric field component along the MP normal (see the negative spike in $E_{x}$ at $\sim 20: 01$ UT in Figs. 1 and 6 and Fig. 9 [A]). This exchange of momentum across the TCS provides effective gyro-viscosity, which automatically results in the magnetic flux diffusion across the MP (see e.g. Sibeck, et al., 1999 and references therein). Another consequence of the exchange by parallel momentum inside the "plasma ball" would be a "pumping" of the inner magnetic field downstream, that enforces the magnetic field minimum over the cusps and, probably, constitutes one of general mechanisms for creating of the large-scale diamagnetic cavities - "plasma balls" (cf. Savin et al., 2005a).

The Poynting vector $\boldsymbol{P}$ (green) in the disturbance at $\sim$ 20:00:40 UT is deflected toward the local normals $N_{E}$ (blue, see [A]) and $N_{B}$ (violet), the $V_{d}$ is close to the model normal at smooth MP $N_{\text {mod }}$ (gray, cf. Cargill et al., 2004). $\boldsymbol{P}$ departs only by 30 degrees from the full velocity of the jet under MP ( $V_{\text {jet }}$, black arrow $)$, further confirming that the jet can irradiate the waves, carrying them downstream; the MP surface charges and/or waves would irradiate disturbances closer to the respective normals $\left(N_{E}, N_{B}\right.$ or $N_{\mathrm{CTA}}-$ dark green, see upper panel of Fig. 5); an exclusion would be a surface wave, moving with group velocity $\sim V_{\text {jet }}$, but in those cases the surface wave is the intermediate means for propagation of the sunward disturbance from the jet towards the MSH.

We showed earlier that the $N_{B}$ and $N_{E}$ normals, obtained from minimum and maximum variance analysis respectively with reasonable eigenvalues (see [A]), differ from each other and especially from the boundary velocities $V_{\mathrm{MP}}$ (red, from constant velocity approach - CVA ( Haaland et al., 2004)), $N_{\text {CTA }}$ and $N_{\text {mod }}$ (see [A] and Cargill et al., 2004). Departure of the "smooth" normal $N_{\text {mod }}$ from the calculated ones is accounted for by the MP indentation over the cusp, which is due to a magnetic field minimum (see Dunlop et al., 2005; Chen et al., 1997; Savin et al., 1998, 2001 and 2004b; Boardsen et al., 2000 and Maynard, 2004), and the respective MP shape is depicted in Insert A of Fig. 5. The normal $N_{E}$ has been calculated for the shortest time interval of 5-6s, and thus it should be defined by the shortest-scale features, including the local normal to the funnel-like cusp throat with the negative $N_{E y}$ and positive $N_{E z}$. Local surface waves can also substantially rotate the MP at those scales (cf. Cargill et al., 2004). However, 54 degrees between $N_{E}$ and $N_{\text {CTA }}$, calculated at the same time scales by different methods (cf. Dunlop et al., 2005), still requires an explanation as the former is repeated on 4 Clusters, and the latter gives the MP thickness, confirmed by the cutoff in ion spectra on Cluster 1 and 3, and is thus fairly reliable. Note that $N_{\text {CTA }}$ is really the direction of the MP velocity, which should hardly change much more than that of $N_{E}$ on different Clusters (i.e. $<10$ degrees). We do not think that such a difference can be uniquely due to a surface wave, as the similar difference ( $\sim 25$ degrees) is between $N_{B}$ and $V_{\text {MP. Dunlop et al. (2005) }}$ for different time intervals obtain $\sim 35$ degrees between $N_{B}$ and their "timing" normal (i.e. CVA). We suggest that the MP movement at the cusp throat, where MP has the complicated shape, cannot be approximated by movement of any plane discontinuity and the MP velocity should not follow the local normal. It means that although the local MP struc- 
ture is well approximated by a plane surface (see also Dunlop et al., 2005), its movement is determined mainly by nonlocal properties of an extremely non-uniform magnetosphere in the outer cusp vicinity. We illustrate the suggestion by a simple example in Insert A of Fig. 5 by taking an indented boundary (lower curve) with violet spots for reference and simulating the MP compression (e.g. due to rising of the SW dynamic pressure) by diminishing its vertical size and enlarging the horizontal one to get the upper curve; then we shift the upper curve vertically until the approximate coincidence of the shift direction of the upper violet spot (violet line) and the local normal (blue line). The latter takes into account the well-known fact that the dayside subsolar MP moves approximately along its normal (see e.g. Sibeck et al., 1999). We do the horizontal enlargement because for the normal being close to X-axis the shift of the boundary depends only on the magnetic field magnitude and its gradient (under MP boundary layer); in the subsolar region (upper part of Insert A) the magnetic field is $>100 \mathrm{nT}$ and it sharply rises inside the magnetosphere, in our case $|B| \sim 30 \mathrm{nT}$ and it first drops just inside the MP (in "plasma ball") and then rises moderately. So, the SW dynamic pressure rising can shift the trailing vertical wall of the cusp much farther than the subsolar MP. This simple consideration conforms to increasing cusp width with the SW dynamic pressure, reported by Van Allen and Adnan (1992) from Hawkeye-1 data. For such boundary transformation, shift vectors of the reference spots in Insert A give a proxy for the MP velocity direction (violet lines). One can see that away from the MP indentation (upper and lower spots) the MP velocities and normals (blue lines) practically coincide, while in the middle the specific movement of the MP as an entity results in the substantial departure of the two directions. Note the MP velocity proxy is close to the average normal $N_{\text {mod. }}$. At the time of the MP encounter by Cluster (see Fig. 5a) with the normal being close to X-axis, the velocity also departs toward $N_{\text {mod. }}$. Thus, an oversimplified deformation model for the indented MP in Insert A provides a hint for explaining the systematic departure of the normal from the MP velocity over cusp, first mentioned by Dunlop et al. (2005).

Returning to the difference of $\sim 16$ degrees between $N_{E}$ and $N_{B}$, the latter is turning towards $N_{\text {mod }}$ as well (Fig. 5a). We account for it by the fact that the normal calculation is influenced less by the MP indentation when it is calculated using data at the larger intervals, in our case $N_{B}$, calculated at $200 \mathrm{~s}$ intervals, corresponding to $\sim R_{E}$ for the measured MP speed, i.e. comparable with the MP indentation with typical scale of 2-3 $R_{E}$ (cf. Savin et al., 1998, 2005a). The same is applicable to $N_{\mathrm{CTA}}$ and $V_{\mathrm{MP}}$ : the latter has been determined from 4-min interval ([A]) and it is much closer to $N_{\text {mod. }}$. As for $N_{\text {CTA }}$, without a respective model we can only suggest similar behavior relative to that of $V_{\mathrm{MP}}$ (i.e. different direction of velocity and normal for a non-planar surface) and keep in mind a possible influence of dynamic behavior of moving MP, including local surface waves. A vector dif- ference $\left(V_{\mathrm{CTA}}-V_{\mathrm{MP}}\right)$ fits a deceleration of the local flow in the large-scale MP frame in the direction normal to the cusp throat wall (Fig. 5a), and to acceleration along the throat boundary (Fig. 5b). The cusp throat appears to move with the average MP as a whole and to interact with local flows at the smaller scales. Note also, that any (originally) plane thin boundary, which moves along its average normal and has superimposed surface waves (either temporal or space ones), should display departures of local normals from the velocity direction. The velocity of the jet inside TCS at 20:0120:02 T, $V_{\text {jet }}$, occurring at 94 degrees to $N_{B}$, departs from the ion velocity in MSH just prior to MP at 20:00 UT, $V_{\mathrm{MSH}}$ (yellow in Fig. 5), in XZ plane slightly towards -Z-axis due to the appearance of $V_{\text {Djet }}$, driven by the negative $E_{x}$ (Fig. 1) in the jet. This backside electric field conforms to a part of the surface charge at the TCS, which is induced by the external flow (see discussion of Insert B in Fig. 1 below); via the cross-field drift the inner plasma is accelerated tailward by the normal electric field, in YZ plane it deflects the flow towards -Y-axis. We assume the latter is a by-product of the induced surface charge.

The clock-angle in the jet at 20:01-20:02 UT change in opposite sense relative to both the inner magnetosphere and GDCF values (cf. Fig. 1 [A] and Insert B in Fig. 1), indicating rotation of the magnetic field to adjust to the electric field, "fixed" at the TCS, similar to that of just outside MP. The same for the $B_{y}$ negative and clock-angle positive departure from their GDCF predictions also in opposite sense relative to the inner magnetosphere 19:56-19:58 UT (cf. Cargill et al., 2004). In fact, one can argue that the jet might be accelerated in the upstream outer cusp, e.g. due to an excess of the plasma pressure there, and both electric and magnetic fields are adjusting to the remote flow and local boundary conditions. But the parallel momentum transfer across the TCS (see Insert B in Fig. 1 and the discussions above) requires generation of the respective local electric field. We have also demonstrated two different ion populations under MP (see Fig. 1 and 7 in [A]), which suggests a combination of local plasma penetration and that of remote one, including possible dayside anti-parallel reconnection (marked by the circled " $r$ " in YZ plane in Fig. 5). We have also mentioned that at the larger scales (see Fig. 1) the positive $E_{x}$ outside MP should be produced by an extra surface charge at MP, which in turn requires the negative $E_{x}$ inside MP (cf. e.g. Nikutowski et al., 1998). This opposite normal electric field inside MP (a consequence of Maxwellian boundary conditions) could have a rather general impact on the MP physics: independent on the origin of the induced electric field outside MP (i.e. independent on the correspondence with GDCF predictions in our case), just inside MP the opposite normal electric field should drive flow perpendicular to the local magnetic field. Thus, the transverse acceleration inside MP boundary layer (usually attributed to reconnection effects, cf. Sibeck et al., 1999) can be an electrodynamic reaction on the external normal electric field. A future task is to check if electron 


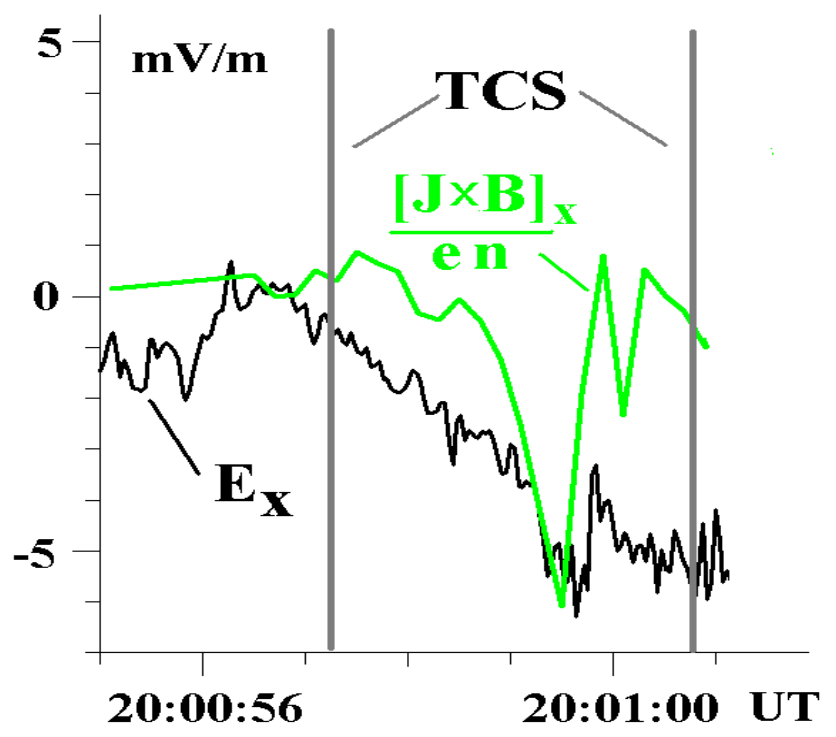

Fig. 6. Comparison of the electric field $E_{x}$ with the Hall term into TCS (see text for details).

field-aligned currents and other kinetic and dynamic effects can neutralize the inner electric field (cf. Genot et al., 2004), especially in the case of a dense inner boundary layer. In this dense layer any necessary "active" surface charge can be easily provided by the field-aligned electrons (with electron density rising by tenths $\mathrm{cm}^{-3}$ ), including that one supporting the non-GDCF positive $E_{x}$, which might serve to remove plasma in front of the moving MP (cf. Fig. 1).

\section{Small-scale electrodynamics}

Another process, with a possible "active" role of the surface charge, is providing the MP current from the Hall current at a TCS of finite-gyroradius scale (required by the magnetic field rotation). The Hall effect results from an electron current due to the cross-field drift of electrons, when a substantial part of the flow-carrying ions move in the direction of electric field (see e.g. Sibeck et al., 1999 and Figs. 2 and 4-6 in [A]). An important issue is that the Hall effect and related thin electron currents are a characteristic feature for any current of ion finite-gyroradius scale independent of reconnection, which certainly should enlarge the mass and momentum transport, but it is not the necessary TCS feature.

Mozer et al. (2003) and Vaivads et al. (2004) provide comprehensive examples primarily for Hall dynamics in TCS from Polar and Cluster data. But they concentrated mostly on a comparison of the data with a limited stationary reconnection model with $\mathrm{X}$-line, requiring a singular "diffusion region". This "diffusion region" should be very limited in space, including its length along MP. In contrast, the ion Hall effect in the thin current sheet should exist all along the sheet, unless the sheet width is comparable with ion gyroradius. In this case the frozen-in condition for ions is violated due to the small current scale, it could be also described in terms of an effective viscosity (see e.g. Stasiewicz, 1994). Belmont and Rezeau, (2001) show similar effects of Alfven waves on the magnetic flux erosion and use the term 'micro-reconnection' in contrast to a stationary laminar reconnection with the Xline configuration (see Sibeck et al., 1999).

Thus we outline the Hall dynamics in the TCS per se, while transient (and multiple) X-, Y-, O-lines etc. might be incorporated into a particular scenario of the current sheet evolution (see e.g. Syrovatskii, 1971; Sibeck et al., 1999; Silin and Buechner, 2003; Savin et al., 2005a). To do a qualitative test particularly for Hall dynamics in the TCS in our case, we calculate the Hall term in the generalized Ohm law (see e.g. Sibeck et al., 1999) using the data from Cluster 1 and inferred TCS geometry and velocity from 4 Clusters and compare its X-component with that of electric field:

$[j \times B]_{x} / e n=-\left(B_{z} d B_{z} / d x+B_{y} d B_{y} / d x\right) / \mu_{0} e n$

where $j$ is the current density, $n$ is the plasma density from spacecraft potential with $5 \mathrm{~Hz}$ sampling, normalized through the WHISPER density data, $e$ is the electron charge. We have neglected all other gradients besides that in X-direction, as the normal $N_{E}$ is close to the $\mathrm{X}$-axis, and the maximum variance procedure gives very different eigen values (i.e. the TCS can be regarded as a locally flat structure, cf. Dunlop et al., 2005, [A]). We also substitute $d x \sim V_{x} d t$, where the MP speed component $V_{x} \sim 30 \mathrm{~km}$ both from $V_{\mathrm{MP}}$ and $V_{\mathrm{CTA}}$ (see [A]). At the distance between spacecraft of $\sim 600 \mathrm{~km}$ with such MP speed one cannot get quantitative estimates of other terms in the $\mathrm{Ohm}$ equation having maximum resolution of the particle instruments of $4 \mathrm{~s}$ (cf. Sibeck et al., 1999; Vaivads et al., 2004; Mozer et al., 2003a). The result of comparison of the main electric component and the Hall term inside TCS is depicted in Fig. 6: the green line shows the Hall-term Xprojection, the black one $E_{x}$; in the middle of the TCS one can see that the spike in $[j \times B]_{x} / e n \sim E_{x}$ (now in the spacecraft frame, unlike Fig. 4), while at its borders the electric field matches the outer values, i.e. the boundary conditions, required for driving the respective frozen-in plasma jets outside the TCS. Thus with the available time resolution we can infer rather space than surface electron charge into the TCS, which serves both to support the necessary separating current via the Hall effect, and to fit the surrounding flows and fields, including the MP motion. Remembering that the variability of magnetic disturbances near the TCS on different spacecraft (Fig. 9 in [A]) introduces extra uncertainty in calculating of the Hall-term; we also take into account the geometrical and single-spacecraft limitations in our calculations of the Hall term. Then we can infer fairly satisfactory fitting of the data to the Hall physics in the center of the TCS. We have checked, that the $E_{x}$ almost does not depend on transformation in frames, moving at speeds $V_{\mathrm{MP}}$ or $V_{\mathrm{CTA}}$ (as $E_{x}$ is close to the normal component, which is not affected by the normal speed of a frame). 
Dunlop et al. (2005) use magnetic data from all spacecraft to calculate the current by the curlometer technique and propose that the magnetic stress $[j \times B]$ at the MP can accelerate the jet just inside the MP downward, i.e. the stress could play an "active" primary role. Our comment on that is that their calculation is made on a $\sim 600 \mathrm{~km}$ scale, which is much larger than that of the TCS width of $\sim 90 \mathrm{~km}$. On the contrary, we propose that this should be a second-order effect, remembering the large ion $\beta$, which is especially high in the "plasma ball" and advocate the decisive role of the electric forces and surface charges. However, we realize that this should be confirmed through an appropriate modeling in future.

The potential difference for driving electron current along field lines can be provided by inertial (polarization) drift of the incident MSH ions in the non-uniform electric field of the surface charge, seen in the frame of bulk motion by the MSH plasma (see Fig. 1, 5, cf. Genot et al., 2004; Savin et al., 2005b). For a non-uniform electric field the zero approximation of the electric field cross-field drift with velocity $V_{d}^{(0)}=[\boldsymbol{E} \times \boldsymbol{B}](\boldsymbol{E}$ and $\boldsymbol{B}-$ electric and magnetic field vectors $)$ is broken and one should take into account the first-order inertial drift (Golant et al., 1977):

$V_{d}^{(1)}=Z e /\left(M \omega_{H}^{2}\right) d \boldsymbol{E} / d t$

where $M, \omega_{H}, Z e$ are respectively the mass, cyclotron frequency and charge of the particles. This approximation is valid only for space scales larger than the ion gyroradius and frequencies smaller than the ion gyrofrequency. In the opposite limit for small scales, the electric field should accelerate the ions directly, providing the Hall electron current in the transverse direction. Regarding the disturbances at 20:00:30-20:00:50 UT in Fig. 4, a characteristic velocity $V_{\text {MP }}(\sim 30 \mathrm{~km} / \mathrm{s}$, ignoring its departure from $\mathrm{X}$-axis) and $|B| \sim 50 \mathrm{nT}$ (see Fig. 1 [A] and 1), one can get the appropriate scaling for different ion energies; the proton gyrofrequency being $\sim 0.75 \mathrm{~Hz}$. As an example we take the $E_{x}^{\prime}$ rise at 20:00:24-26:00 UT (see Fig. 4), then (2) gives $V_{d}^{(1)} \sim 3 \mathrm{~km} / \mathrm{s}$; the respective maximum ion shift from electrons in $\mathrm{X}$ direction due to inertial drift $L_{i} \sim 5 \mathrm{~km}$ (i.e. of the order of few electron inertial lengths or that of gyroradii); the inferred structure scale $d_{p} \sim 50 \mathrm{~km}$ that corresponds to gyroradius of $\sim 500 \mathrm{eV}$ protons, so that the drift approximation is valid only for the protons with smaller energy. The protons with higher energies should loose $\sim 100 \mathrm{eV}$ in the sunward directed electric-field pulse of finite-gyroradius scale. In another spike at 20:00:15 UT with the inferred width of $\sim 60 \mathrm{~km}$ and total potential change of the order of $180 \mathrm{~V}$, the inertial drift effects are negligible as the spike switches on and off faster than proton gyroperiod. Protons with energy $>900 \mathrm{eV}$ (gyroradius $\sim 60 \mathrm{~km}$ ) loose the total potential energy; that of $\sim 200 \mathrm{eV}$ (and less) with gyroradius of the order of a half of the pulse width do not change their energies as the loss at the leading front is comparable with the gain at the trailing one. From 200 to $900 \mathrm{eV}$ the energy loss rises to $180 \mathrm{eV}$, so that the respective velocity dispersion should result in a space charge, presumably neutralized by the parallel electron currents (cf. Genot et al., 2004). This inferred interaction with the small-scale electric fields is similar to that of the Hall dynamics of the TCS, discussed in section "Plasma inflow and momentum balance". While some electric bursts, to which we attribute potentials $\sim 100 \mathrm{~V}$, could be temporary, the high correlation of the $E_{x}$-signals (especially $\sim 0.93$ on Clusters 2-4, separations of which in the normal direction is comparable with the TCS width, see [A]) with the definite lags, suggests their spatial nature in the majority of cases.

During the strong sunward electric field from 19:55 to 20:00 UT (see Fig. 7), the higher-energy protons meet several positive $E_{x}^{\prime}$-pulses, thus loosing several hundreds $\mathrm{eV}$. As we have seen (Fig. 2), the ions with energy $>300 \mathrm{eV}$ carry main the flow near MP, so namely those ions are subjected to decelerating by the spikes of the normal electric field. This interaction could also account for the density rises of the higher-energy ions (due to piling up by the electric structures), correlating with the $E_{x}$-disturbances, in Fig. 1 after the disturbance in electric field $\sim$ at 19:50 UT.

The velocity of inertial drift changes sign at positive and negative $d E_{x} / d t$ (see Eq. 2), being of the order of few $\mathrm{km} / \mathrm{s}$ (maximum), i.e. its influence on the ion dynamics is negligible. Typical shift of ions from electrons due to the drift is $5-50 \mathrm{~km}$, that infers a space charge at the edges of the smooth gradients in electric field with characteristic potential difference of several tens of volts (of the order of the change of potential at these distances in the measured fields). Genot et al. (2004) simulated similar features of the inertial drift in a non-uniform electric field of a finite-amplitude Alfven wave, and have demonstrated that the ion space charge is neutralized by parallel electron currents, splitted into thin layers of electron-inertial size. Our characteristic potential differences are similar to that of the simulations, and possible energy gains in them can exceed the electron temperature. The latter should result in electron beams at superthermal speeds, which in turn generate high-frequency waves up to plasma frequency; similar waves are seen in WHISPER data on the top panel in Fig. 6, including trailing TCS edge $(\sim 20-60 \mathrm{kHz})$. Correlation between positive $E_{x}^{\prime}$ spikes (in the MSH frame, panel b) and the plasma waves (20-60 kHz in top panel) is not very high: only $\sim 56 \%$ of the spikes with $E_{x}^{\prime}>5.5 \mathrm{mV} / \mathrm{m}$ can be associated at the spacecraft spin resolution with the wave bursts, and $\sim 55 \%$ wave bursts - with $E_{x}^{\prime}>4.5 \mathrm{mV} / \mathrm{m}$. But it could be fairly satisfactory, taking into account that the parallel electron flows are defined by potential difference along particular field lines and by the neutralization current speed being larger or smaller than the thermal electron one, i.e. they have nonlocal character in contrast to the local electric field measurements. Another experimental support for the suggestion of the plasma-wave generation by the disturbed thin electron sheets follows from comparison of the top and bottom panels in Fig. 7. The panel c displays 

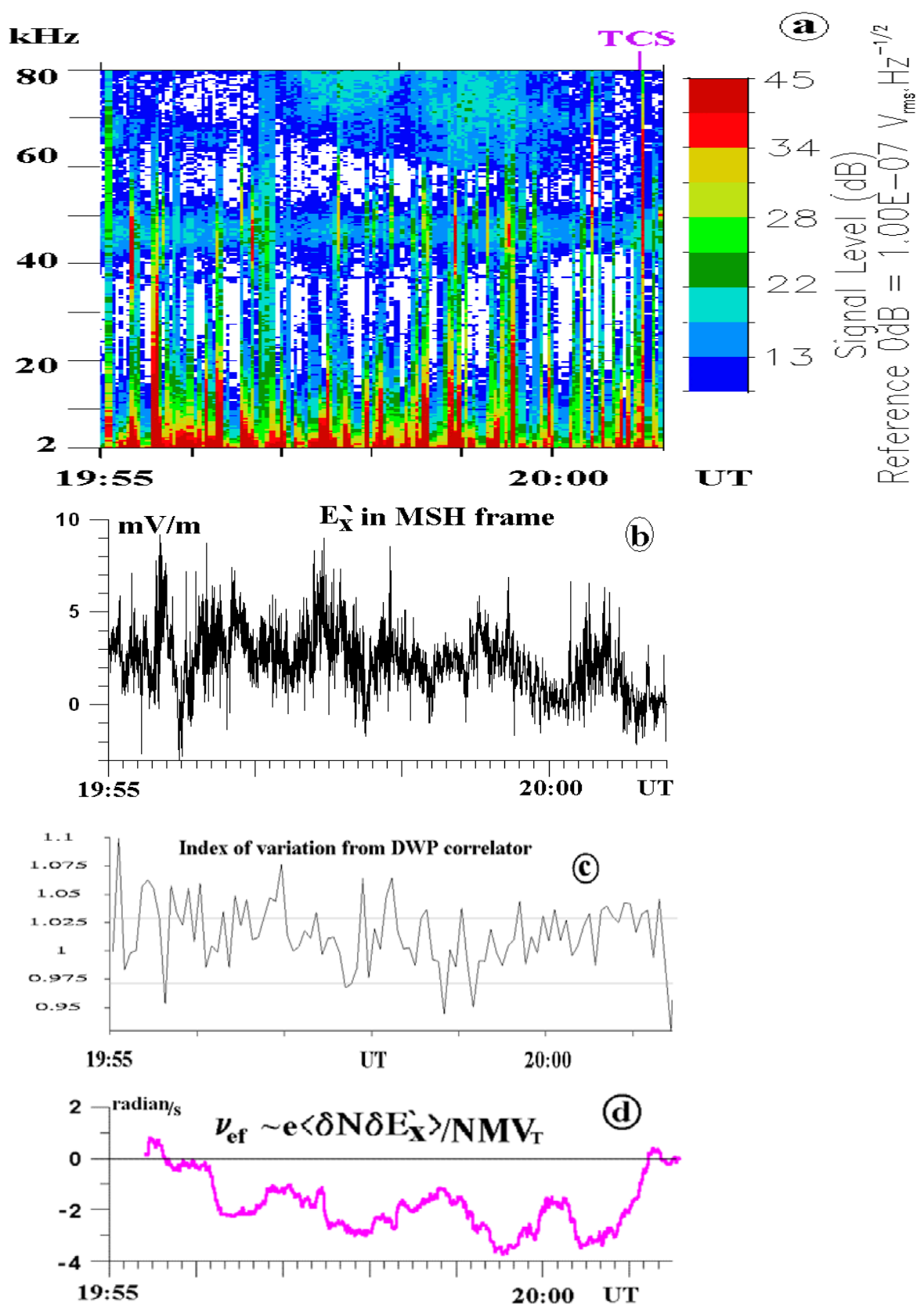

Fig. 7. Cluster-1, 13 February 2001. (a) WHISPER electric field spectrogram (see [A]) with color-coded intensity (scale at the right side); (b) electric field $E_{x}^{\prime}$ in the MSH frame are superimposed (see Fig. 5); (c) index of variation as deduced from the DWP correlator measurements for the pre-set energy (i.e. $40 \mathrm{eV}$ to $100 \mathrm{eV}$ ), which quantifies the variance in the electron counting process that the DWP correlator performs. The values are normalized to the electron count rate and a value of unity indicates the electrons are random Poisson distributed; (d) an estimate of ion effective collision frequency, deduced from correlated fluctuations of ion density and $E_{x}^{\prime}$ for the band $0.1-2.5 \mathrm{~Hz}$, averaged over $50 \mathrm{~s}$.

an index of variation as deduced from the Data Wave Processor (DWP, see Woolliscroft et al., 1997) correlator measurements for the pre-set energy $40 \mathrm{eV}$ to $100 \mathrm{eV}$. The index of variation quantifies the variance in the electron counting process; the values are normalized to the estimated count rate and a value of unity indicates the electrons are random
Poisson distributed (i.e. undisturbed). Disturbed electron distributions include fluctuating electron beams, similar to the model ones (see Genot et al., 2004) and indicate substantial AC disturbances in electron current. While the index of variations infers the presence of a variety of different presumably non-linear disturbances, up to now it is the only 
electron parameter, which provides information about local processes in the high-frequency range at electron-scales. In any case, the index displays non-equilibrium state of electrons. In Fig. 7c we put a threshold of $+/-0.03$ for the index of variations, marked by horizontal lines, and in $\sim 73 \%$ cases at the time scale of spacecraft spin one can see appearance of wave spikes on the top panel in the band of the electron plasma frequency $(20-60 \mathrm{kHz})$; the reverse cross-correlation is $\sim 53 \%$. A narrowness of the electron disturbances follows from the correlator sample group interval of 732 microseconds, which corresponds to $20 \mathrm{~m}$ (of the order of the Debye length) in the case of scaling by the MP speed of $\sim 30 \mathrm{~km} / \mathrm{s}$, and to $200 \mathrm{~m}$ for that of the MP jet (see Fig. 1 [A]). The scales of electron disturbances look to be underestimated on the background of electron inertial length and gyroradius being of few $\mathrm{km}$, but certainly these scales are much shorter than the characteristic ion ones. The similarity in behavior of the high-frequency waves in electric field and the electron index of variation is suggestive for the local origin of the waves and/or their strong interaction with the electrons.

To get an order-of-magnitude estimate for the effective collision frequency $\left(v_{e f}\right)$ of the ions with the electric fluctuations, we calculate in $10 \mathrm{~s}$ intervals and average over 50 $\mathrm{s}$ the value of the product of the plasma density $(\delta N)$ and electric fluctuations in the MSH frame $\left(\delta E_{x}^{\prime}\right)$ in frequency band $0.1-2.5 \mathrm{~Hz}$, using spacecraft potential with $5 \mathrm{~Hz}$ sampling as a proxy for the density measurement after its recalibration by the WHISPER plasma density data. Figure 7d demonstrates that further averaging will not change qualitatively the pattern: in the region of the inferred interactions the electric field bursts anti-correlate with those of the density, presumably suggesting effective collisions. We have done re-calculation of the averaged $\delta N \delta E_{x}^{\prime}$ into the effective frequency scale similar to Panov et al. (2005). To that purpose, we consider a reduced equation of ion motion in the MSH flow frame and equalize the ion momentum change due to fluctuations by the averaged nonlinear term:

$N M d V_{x} / d t \approx N M V_{i T} v_{e f} \approx e\left\langle\delta N \delta E_{x}^{\prime}\right\rangle$

We find that

$v_{e f} \approx e\left\langle\delta N \delta E_{x}^{\prime}\right\rangle / N M V_{i T}$

where $M, V_{i T}$ - proton mass and thermal velocity.

Taking $V_{i T}$ as a proxy for the characteristic velocity poses its maximum value, that gives minimal estimate for the $v_{e f} \sim 2$ radian/s in the interaction region in the middle of Fig. 7d. This $v_{e f}$ is comparable with the proton gyrofrequency $(\sim 0.75 \mathrm{~Hz})$, that infers Hall dynamics in this region, similar to that of the thin MP. $v_{e f}$ becomes smaller if one takes higher frequency limit for filtering $\delta N$ and $\delta E_{x}^{\prime}$ before calculating (3), but qualitatively the latter suggestion holds.

So, our considerations suggest that in front of MP the sunward electric field in the MSH frame: (i) by its DC component rotates the magnetic field and accelerates the flow down- stream along the MP; (ii) by its spikes and gradients decelerates the higher-energy ions and produce space charges, most probably neutralized by parallel electron flows. Thus, we propose that the smaller-scale electric fields provide a kind of effective collision process for decreasing the average normal flows in the MP vicinity. Equalizing of the parallel and perpendicular ion temperatures in the zones of intensive electric fluctuations (Fig. 1 [A]) along with the temperature rise in front of MP, conforms to the hypothesis of effective collision operations. Another consequence of the effective collisions should be the heating of $\mathrm{O}+$ from the outer magnetosphere, where its larger gyroradius should provide the more effective interaction with the short-scale electric bursts, compared to protons. In the presence of a surface charge $\mathrm{O}+$ should be accelerated while moving in the direction of the DC electric field outward; we will discuss those points in details in a future paper.

\section{Discussion and conclusions}

The Hall effect at such thin charged layers, as the TCS discussed above, separating different plasmas, can serve to support the transverse current between the plasmas selfconsistently (i.e. without any "anomalous" resistivity or a "diffusion region"). It does not necessarily imply macro reconnection with parallel electric field, while reconnection should include the ion-scale layers. In other words, a charged TCS just because of its ion-gyroradius width becomes partially transparent for the larger-energy ions and respective magnetic flux without any extra change of the field topology (which could be superimposed or not). Transport of momentum across the layers is also provided by ions with larger gyroradius without any magnetic field annihilation. The momentum transfer has been discussed by Stasiewicz (1994) in terms of gyro-viscosity, which in the case of anti-parallel magnetic fields predicts a forcing of the boundary Earthward without a macro-reconnection. The ratio of the normal to MP gyroviscous stress to Maxwell stresses is $\sim \beta \rho_{u} / 2 d$, where $\rho_{u}$ is directional gyroradius and $d-$ the MP width. Over the cusp and especially at the boundary of "plasma balls" both $\beta$ and $\rho_{u}$ are rising due to the magnetic field minimum and due to the acceleration of the flow around MP towards the tail; thus, the MP inward motion due to this effect should have a maximum at the sunward edge of the cusp for the IMF $B_{z}<0$ in our case (while Cluster entered the cusp at its tailward edge). In spite of sampling of the MP outside the maximum gyro-viscosity effect, Fig. 2 demonstrates clear momentum transfer across the MP, especially in its component parallel to MP.

We have discussed that the ion inertial drift in the electric fields at TCS can result in charge separation, probably neutralized by parallel electron currents, and the instability of which produces transverse electron scales (cf. Genot et al., 2004). In this case, the cascading towards electron scales 
should provide a similar measurement pattern, as supposed, but never clearly demonstrated, for the "diffusion region" of macro reconnection. So, we are skeptical about recent "discoveries" of the "diffusion regions" (see e.g. Mozer et al., 2003a; Vaivads et al., 2004; and references therein): the electron scales are mandatory for the localized "diffusion region", but their presence all along a thin MP does not necessarily imply the boundary topology change and release of magnetic energy, stored in the deformed magnetic field (i.e. a macro reconnection). Mozer et al. (2003) concluded that "significant conversion of electromagnetic energy can occur inside the magnetopause in the absence of an electron diffusion region, parallel electric fields, or the electrons being decoupled from the magnetic field. ... these properties are consequences of the Hall MHD and guide electric and magnetic fields in the absence of any additional non-MHD processes". In this paper we are outlining the effects of electric fields at the finite-gyroradius scales, which should accompany any Hall-MHD structures (with scales of the order of the ion gyroradius of, at least, the higher-energy ions, carrying nevertheless the substantial part of the plasma momentum). We plan to check our suggestion of the appearance of electrons scaled as a consequence of the inertial drift by respective modeling in a future paper.

In $[\mathrm{A}]$ we demonstrate the existence in the MP vicinity of at least three ion sub-populations from different sources, including mantle-like ions, which are usually attributed to the remote reconnection on dayside (see Fig. 5 and related discussions above). Outlining here the operation of the local finite-gyroradius penetration and MP charging, we would be unable to define a mutual importance of the local and remote mechanisms for plasma transport and jet formation, basing only on the local measurements. Gas dynamic modeling with realistic MP shape, obtained from the pressure balance, promises to shed a light on that in a future paper. A brief inspection of the electron data (see Fig. 3 in $[\mathrm{A}]$ and related discussions) did not improve much the situation: the counterstreaming heated and core populations can be accounted for the dayside reconnection, while the cold anti-parallel electrons (i.e. flowing from the tail towards the dayside along the MSH field lines near MP) are detected throughout MSH, starting from a postshock region at 16:50 UT (not shown, cf. anti-parallel flows at 50-77 eV in Fig. 3 from [A]). Because of that these electrons hardly can be used in our case as the reconnection tracers. As for the bi-directional electrons outside MP, Savin et al. (2004b) accounted for their heating near MP by positioning spacecraft inside the distributed region of electron acceleration by presumably waves in the low-hybrid band. Dominating of the parallel electrons is a hint for a superimposed distant source, first of all of the dayside reconnection site. Appearance of the quasi-perpendicular electrons at the higher energies (cf. Fig. 3 in [A]) can be attributed to the fan instability of the parallel electron flows (see Savin et al., 1997, 1998 and references therein). Taking 10 periods of the low hybrid frequency $(10-20 \mathrm{~Hz})$ as a proxy for the time of fan instability development, for the velocity of $100 \mathrm{eV}$ electrons one would get a distance to a source of $\sim 0.5-1 R_{E}$, that corresponds rather to ion time-offlight estimates (that gives the spread of the finite-gyroradius penetration of $2-3 R_{E}$, see Fig. 3 and related discussions), than to the distance from the dayside reconnection site (cf. Fig. 5b). The time-of-flight of the bursty protons, leaking from the outer cusp presumably via finite-gyroradius effect, can be utilized for remote sounding of the MP width along field lines.

Finally, the study of nonlinear dynamics of the plasma flow interaction with the geomagnetic trap highlights a fundamental role of the finite-gyroradius effects, surface charges and related accelerated plasma jets. In the MP boundary layers the accelerated jets provide a flow balance via the rotation of magnetic field (relative to GDCF model predictions) and via the "active" electric field (superimposed on that predicted by GDCF model), most probably supported at the moving and indented MP by parallel electron currents. The complicated MP shape suggests its systematic velocity departure from the local normal towards the average one. The electric fields in the MSH frame both accelerate the MSH plasma along MP downstream and provide effective collisions via deceleration of the higher-energy MSH ions in the process of interaction with the finite-gyroradius electric bursts. These interactions should reduce the MSH normal flows just in front of MP and create charge separation, which is suggested to be neutralized by parallel electron currents. The effective collisions, with frequencies of the order of the ion cyclotron one as estimated from the nonlinear transport term in (3), should result in the ion heating (cf. Fig. 1 in [A]) and in the Hall ion dynamics, i.e. acquisition of the larger-scale transverse electric potentials (due to quasi-DC electric field in the MSH frame) by the higher energy ions. While our order of magnitude calculations of the collision frequency do not provide quantitative estimates for the ion dynamics, yet they outline the valuable role of the nonlinear correlations (i.e. the finite average value of $\delta N \delta E_{x}^{\prime}$ ), which should be taken into account in the problem treatment and modeling. Based on the above considerations, we are preparing the separate paper on comparison of this data with nonlinear turbulence modeling in the presence of the DC electric field. These effective collisions in the extended turbulent zones (cf. Savin et al., 1998, 2002, 2004b) are a promising alternative in place of the usual parallel electric fields invoked in the macro-reconnection scenarios.

Acknowledgements. Work was partially supported by INTAS03-50-4872, INTAS 05-1000008-8050, RFFS 06-02-17256, 04-02-17371 and 03-02-16967, ISSI (Team 81), KBN 8T12 E 016 28, ECRT Network HPRN-CT-2001-0314, and Science School 173920032.

Edited by: N. Watkins

Reviewed by: C. Watt and another referee 


\section{References}

Amata, E., Savin, S., Andre, M., Dunlop, M., Marcucci, M. F., et al.: Experimental study of nonlinear interaction of plasma flow with charged thin current sheets: 1 . Boundary structure and motion, Nonlin. Processes Geophys., 13, 365-376, 2006 (cited as [A]).

Andre, M., Vaivads, A., Buchert, S. C., Fazakerley, A. N., and Lahiff, A.: Thin electron-scale layers at the magnetopause, Geophys. Res. Lett., 31, L03803, doi:10.1029/2003GL018137, 2004.

Belmont, G. and Rezeau, L.: Magnetopause reconnection induced by Hall-MHD fluctuations, J. Geophys. Res., 106(A6), 10 75110760, 2001.

Boardsen, S. A., Eastman, T. E., Sotirelis, T., and Green, J. L.: An empirical model of the high-latitude magnetopause, J. Geophys. Res., 105, 23 193-23 220, 2000.

Cargill, P. J., Dunlop, M. W., Lavraud, B., Elphic, R. C., Holland, D. L., et al.: CLUSTER encounters with the high altitude cusp: boundary structure and magnetic field depletions, Ann. Geophys., 22, 1739-1754, 2004,

http://www.ann-geophys.net/22/1739/2004/.

Chen, S.-H., Boardsen, S. A., Fung, S. F., Green, J. L., Kessel, R. L., Tan, L. C., Eastman, T. E., and Craven,J. D.: Exterior and interior polar cusps: Observations from Hawkeye, J. Geophys. Res., 102(A6), 11 335-11348, 1997.

Dunlop, M. W., Lavraud, B., Cargill, P., Taylor, M. G. T., Balogh, A., et al.: Cluster observations of the cusp: magnetic structure and dynamics, Surveys in Geophys., 26, 5-55, 2005.

Genot, V., Louarn, P., and Mottez, F.: Alfven wave interaction with inhomogeneous plasmas: acceleration and energy cascade towards small-scales, Ann. Geophys., 22, 2081-2096, 2004, http://www.ann-geophys.net/22/2081/2004/.

Golant, V. E., Zhilinski, A. P., and Sakharov, S. A.: Basic Plasma Physics, Atomizdat, 245-248, 1977.

Haaland, S. E., Sonnerup, B. U. "O., Dunlop, M. W., Balogh, A., Georgescu, E., et al.: Four-spacecraft determination of magnetopause orientation, motion and thickness: comparison with results from single-spacecraft methods, Ann. Geophys., 22, 13471365, 2004, http://www.ann-geophys.net/22/1347/2004/.

Haerendel, G.: Microscopic plasma processes related to reconnection, J. Atmos. Terr. Phys., 40, 343-353, 1978.

Lavraud, B., Dunlop, M. W., Phan, T. D., Reme, H., Bosqued, J. M., et al.: Cluster observations of the exterior cusp and its surrounding boundaries Under northward IMF, Geophys. Res. Lett., 29, 20, 1995, doi:10.1029/2002GL015464, 2002.

Lavraud, B., Reme, H., Dunlop, M. W., Bosqued, J., Dandouras, I., et al.: Cluster observes the high altitude/exterior cusp regions, Surveys in Geophys., 26, 1-3, 135-175, 2005.

Maynard, N. C.: Coupling of the solar-wind/IMF to the ionosphere through the high latitude cusps, Surveys in Geophys., 26, 1-3, 255-280, 2005.

Mozer, F. S., Phan, T. D., and Bale, S. D.: The complex structure of the reconnecting magnetopause, Phys. Plasmas, 110, 2480, doi:10.1063/1.1570419, 2003.

Mozer, F. S., Bale, S. D., Phan, T. D., and Osborne, J. A.: Observations of Electron Diffusion Regions at the Subsolar Magnetopause, Phys. Rev. Lett., 91, 245002, doi:10.1103/PhysRevLett.91.245002, 2003a.

Nikutowski, B., Buchner, J., Wiechen, H., Auster, U., Fornacon,
K. H., Rustenbach, J., Klimov, S., and Savin, S.: A highlatitude boundary layer crossing - INTERBALL measurements and MHD model results, Adv. Space Res., 22, N1, 161-165, 1998.

Nikutowski B., Buechner, J., Klimov, S., Petrukovich, A., Romanov, S., and Savin, S.: INTERBALL observations of field aligned current signatures due to collisionless reconnection, in: VIIth International Conference on Plasma Astrophysics and Space Physics, edited by: Buchner, J., Axford, I., Marsch, E., Vasyliunas, V., Kluwer AP, Dordrecht, 687-692, 1999.

Panov, E., Buechner, J., Franz, M., Korth, A., Khotyaintsev, Y., et al.: CLUSTER spacecraft observation of a thin current sheet at the Earth's magnetopause, Adv. Space Res., 37, 1363-1372, 2006.

Savin, S. P., Balan, O., Borodkova, N., Budnik, E., Nikolaeva, N., et. al.: Interball Magnetotail Boundary Case Studies, Adv. Space Res., 20, 4-5, 999-1015, 1997.

Savin, S. P., Borodkova, N. L., Budnik, E. Yu., Fedorov, A. O., Klimov, S. I., et al.: Interball tail probe measurements in outer cusp and boundary layers, in: Geospace Mass and Energy Flow: Results from the International Solar-Terrestrial Physics Program, edited by: Horwitz, J. L., Gallagher, D. L., and Peterson, W. K., American Geophysical Union, Washington D.C., Geophysical Monograph, 104, 25-44, 1998.

Savin, S. P., Zelenyi, L. M., Romanov, S. A., Klimov, S. I., Skalsky, A. A., et al.: Turbulent Boundary layer at the Border of Geomagnetic Trap, JETP Letters, 74, 11, 547-551, 2001.

Savin, S., Buechner, J., Consolini, G., Nikutowski, B., Zelenyi, L., Amata, E., et al.: On the properties of turbulent boundary layer over polar cusps, Nonlin. Processes Geophys., 9, 443-451, 2002, http://www.nonlin-processes-geophys.net/9/443/2002/.

Savin, S., Zelenyi, L. M., Amata, E., et al.: Dynamic Interaction of Plasma Flow with Hot Boundary Layer of Geomagnetic Trap, JETP Letters, 79, 452-456, 2004a.

Savin, S., Zelenyi, L., Romanov, S., Sandahl, I., Pickett, J., Amata, E., et al.: Magnetosheath - Cusp Interface, Ann. Geophys., 22, 183-212, 2004b.

Savin, S., Skalsky, A., Zelenyi, L. Song, P., Fritz, T. A., Amata, E., Buechner, J., Blecki, J., et al.: Magnetosheath interaction with high latitude magnetopause, Surveys in Geophys., 26, 95-133, 2005a.

Savin, S., Zelenyi, L., Amata, E., Buechner, J., Blecki, J., Greco, A., Klimov, S., Lopez, R. E., Nikutowski, B., Panov, E., et al.: Magnetosheath interaction with high latitude magnetopause: dynamic flow chaotization, Planet. Space Sci., 53, 133-140, 2005b.

Scudder, J. D., Mozer, F. S., Maynard, N. C., and Russell, C. T.: Fingerprints of collision-less reconnection at the separator, I, Ambipolar-Hall signatures, J. Geophys. Res., 107(A10), 1294, doi:10.1029/2001JA000126, 2002.

Sibeck, D. G., Paschmann, G., Treumann, R. A., Fuselier, S. A., Lennartsson, O. W., et al.: Plasma Transfer Processes at the Magnetopause, Space Sci. Rev., 88, 207-283, 1999.

Silin, I. and Buechner, J.: Nonlinear instability of thin current sheets in antiparallel and guided magnetic fields, Phys. Plasma, 10, 9, 1-10, 2003.

Song, P., Russell, C. T., Gombosi, T. I., Spreiter, J. R., Stahara, S. S., and Zhang, X. X.: On the processes in the terrestrial magnetosheath, 1: Scheme development, J. Geophys. Res., 104, 22 345-22 356, 1999. 
Stasiewicz, K.: Finite Larmor radius effects in the magnetosphere, Space Sci. Rev., 65, 221-253, 1994.

Syrovatskii, S. I.: Formation of current sheets in a plasma with a frozen-in strong magnetic field, Sov. Phys. JETP, 33, 933-940, 1971.

Taktakishvili , A., Greco, A., Zimbardo, G., Veltri, P., Cimino, G., Zelenyi, L., and Lopez, R. E.: The penetration of ions into the magnetosphere through the magnetopause turbulent current sheet, Ann. Geophys., 21, 1965-1973, 2003, http://www.ann-geophys.net/21/1965/2003/.

Vaivads, A., Khotyaintsev, Y., Andre, M., Retino, A., Buchert, et al.: Structure of the Magnetic Reconnection Diffusion Region from Four-Spacecraft Observations, Phys. Rev. Lett., 93, 10, 105001, doi:10.1103/PhysRevLett.93.105001, 2004.

Van Allen, J. A. and Adnan, J.: Observed currents on the earth's high-latitude magnetopause, J. Geophys. Res., 97, 6381-6395, 1992.
Wang, Y. L., Raeder, J., and Russell, C. T.: Plasma depletion layer: Magnetosheath flow structure and forces, Ann. Geophys., 22, 1001-1017, 2004, http://www.ann-geophys.net/22/1001/2004/.

Woolliscroft, L. J. C., Alleyne, H. St. C., Dunford, C. M., Sumner, A., Thomson, J. A., Walker, S. N., Yearby, K. H., Buckley, A., Chapman, S., Gough, M. P., and the DWP co-investigators: The Digital Wave-Processing Experiment on Cluster, Space Sci. Rev., 97, 209-231, 1997.

Zelenyi, L. M., Delcourt, D. C., Malova H. V., and Sharma, A. S.: "Aging" of the magnetotail thin current sheets, Geophys. Res. Lett., 29, 12, doi:10.1029/2001GL013789, 2002. 